\title{
Effect of Persian Medicine Remedy on Chemotherapy Induced Nausea and Vomiting in Breast Cancer: A Double Blind, Randomized, Crossover Clinical Trial
}

\author{
Mohammad Nazari ${ }^{1}$, Ali Taghizadeh², Mojtaba Mousavi Bazzaz ${ }^{3}$, Hassan Rakhshandeh ${ }^{4}$, Sadegh Shokri ${ }^{5}$ \\ ${ }^{1}$ Ph.D. of Persian Medicine, Assistant Professor, Department of Persian medicine, School of Persian and \\ Complementary Medicine, Mashhad University of Medical Sciences, Mashhad, Iran \\ ${ }^{2}$ Associate Professor, Surgical Oncology Research Center, Mashhad University of Medical Sciences, Mashhad, Iran \\ ${ }^{3}$ Associate Professor, Department of Community Medicine, School of Medicine, Mashhad University of Medical \\ Sciences, Mashhad, Iran \\ ${ }^{4}$ Assistant Professor, Pharmacological Research Center of Medicinal Plants, Mashhad University of Medical \\ Sciences, Mashhad, Iran \\ ${ }^{5}$ Ph.D. of Persian Medicine, School of Persian and Complementary Medicine, Mashhad University of Medical \\ Sciences, Mashhad, Iran
}

Type of article: Original

\begin{abstract}
Background: Chemotherapy induced nausea and vomiting (CINV) is a side effect, and has negative effect on quality of life and continuation of chemotherapy. Despite new regimen and drugs, the problems still remain and standard guidelines, effective treatment and supportive care for refractory CINV are still not yet established. Persian medicine, the old Iranian medical school, offer Persumac (prepared from Rhus Coriaria and Bunium Persicum Boiss).

Objective: The specific objectives were to assess the effect of Persumac on the number and severity of nausea and vomiting in refractory CINV in acute and delayed phase.

Methods: This randomized, double blind, crossover clinical trial study was carried out on 93 patients with breast cancer and refractory CINV, who received outpatient high emetogenic chemotherapy in Imam Reza hospital, Mashhad, Iran from October 2015 to May 2016. The study has three stages: in stage I patients received a questionaire and completed it after chemotherapy. In stage II they were randomly divided into intervention group with Persumac and control group with placebo (lactose were used). In stage III, wash out and crossover was conducted. Both groups in all stages received standard antiemetic therapy for CINV. The following were set as the inclusion criteria of the study: female, Age $\geq 18$ years, clinical diagnosis of breast cancer, history of refractory CINV, normal blood tests and at least three courses of chemotherapy remaining. Exclusion criteria of this study were: Total or upper abdominal radiation therapy along with chemotherapy, drugs/therapy for nausea and vomiting not prescribed in this study, hypersensitivity to Sumac or Bunium Persicum, use of sumac and Bunium Persicum in seven days prior to the intervention, clinical diagnosis of digestion disorders, non-chemotherapy induced nausea and vomiting, milk allergy, loss of two consecutive or three intermittent doses of Persumac or placebo. Outcomes were gathered by Persian questionnaire. Number and severity of nausea and vomiting was measured with a self-reporting tool; visual analog scale.

Results: Demographic data and other characters in both groups have no significant diffrence. Eighty of 93 eligible patients in stage I completed the study and in stage II, eleven declined participation for stage III (crossover). P value of carry over, period and treatment effects demonstrated that they had not affected the results before and after crossover. The mean severity of nausea in acute phase was in stage I: $4.83 \pm 1.40$, stage II: $4.54 \pm$ 2.0 and stage III: $4.15 \pm 0.92$ in sequence $\mathrm{AB}$ (first Persumac and then placebo in crossover), and in sequence BA (first placebo and then Persumac in crossover) was respectively $4.83 \pm 1.40,4.54 \pm 2.0,4.15 \pm 0.92$ with $p$ value of carry over effect: 0.03 and period effect: 0.22 . Except for severity of nausea in acute phase, the mean number
\end{abstract}

\section{Corresponding author:}

Dr. Sadegh Shokri, School of Persian and Complementary Medicine, Mashhad University of Medical Sciences, Mashhad, Iran. Tel: +98.5138552188, Fax:+98.5138535980, E-mail: Shokris1@mums.ac.ir

Received: July 03, 2016, Accepted: November 26, 2016, Published: January 2017

iThenticate screening: November 23, 2016, English editing: December 18, 2016, Quality control: January 06, 2017

(C) 2017 The Authors. This is an open access article under the terms of the Creative Commons Attribution-NonCommercialNoDerivs License, which permits use and distribution in any medium, provided the original work is properly cited, the use is non-commercial and no modifications or adaptations are made. 
and severity of nausea and vomiting scores significantly decreased in acute and delayed phase of CINV.

Conclusion: Persumac may control the refractory CINV. The implicable and clinical importance of this research is that another option exists for refractory CINV. Higher doses, different cancers, patients with more various features, and more complete methodology and tools can provide appropriate designs for new research on this topic.

Trial registration: This trial was registered at the Clinical Trials.gov ID: NCT02787707.

Funding: This study is part of a Ph.D. thesis and under grant; No: 930735 of Research Chancellery of MUMS.

Keywords: Breast cancer, Traditional medicine, Chemotherapy Induced Nausea and Vomiting (CINV), Rhus Coriaria, Bunium Persicum

\section{Introduction}

Chemotherapy Induced Nausea and Vomiting (CINV) has negative effect on patients' quality of life. In addition to problems such as loss of appetite, weakness, weight loss, electrolyte imbalances, dehydratation which can induce fractures, esophagous rupture, psychologic disorders and wound dehiscence (1). Despite the new drugs' findings and achievements on the mechanism of action, thirty percent of patients still experience CINV and the problem still needs to be controlled. (2). Guidelines, effective treatment and supportive measures, and the general consensus about ineffective standard methods of controlling refractory CINV still does not exist (3) This has prompted researchers to explore new methods, treatments and guidelines. (4). When the disease is not controlled well in conventional medicine, patients tend to refer to other medical schools such as complementary and alternative medicine (CAM), including Iranian traditional medicine or Persian medicine. This could be because they believe there are less side effects and costs, better treatment and easy access. Due to the limited effects and the common side effects of antiemetics such as headaches, transient increase in transaminases, constipation and increased risk of fatal torsades de points and in neurokinins (NK-1) with side effects such interference with warfarin and oral contraceptive pills, the early use of traditional medicine is safe. For decades, these therapies were used worldwide among patients, their families and health personnel. These methods are used in 50-84\% of patients with cancer (highest use in breast cancer) $(5,6)$. Iranian traditional medicine or Persian medicine has its own approach to diseases. The ancient Iranian physicians (such as Hakeem) believed that nausea and vomiting had gastric origin in functional view. Stomach distress by various factors such as inappropriate humors production or pouring abnormal materials from part or all of the body can cause nausea and vomiting. According to the etiology and mechanism of disease, treatment methods were mentioned. One of them is Persumac; a combination of Rhus Coriaria and Bunium Persicum that have effect on nausea and vomiting induced by inappropriate humors especially safra (yellow bile) humor $(7,8)$. The general objective of this research is the effect of Persumac in refractory CINV. The specific objectives were to assess the effect of Persumac on number and severity of nausea and vomiting in acute and delayed phase of CINV.

\section{Material and Methods}

\subsection{Research design}

The study is a double blind, randomized, crossover clinical trial on breast cancer patients candidated for chemotherapy. Sampling period was from October 2015 to May 2016.

\subsection{Sampling}

Study population was female breast cancer patients, referred to the oncology clinic at the Imam Reza Hospital affiliated to Mashhad University of Medical Sciences (MUMS), a referral center in northeast Iran. Patients with a definite breast cancer diagnosis by pathologists and oncologist, with each type of pathology and grade, were recruited, and according to standard protocols, candidates for chemotherapy with moderate or high emetogenic risk: Adriamycin + cyclophosphamide (AC) + paclitaxel dose dense every 2 weeks 8 courses, and Ac + paclitaxel every 3 weeks 8 courses (2) .and despite standard CINV therapy (Aprepitant, granisetron and dexamethasone), the patients' problems persisted.

\subsubsection{Sample size}

According to $70 \%$ effect of standard CINV therapy; P1 $=70 \%$ (2) and estimation of impact of Persumac on CINV above $90 \%$ based on unpublished pilot study by authors; $\mathrm{P}_{2}=90 \%$ and alpha error of $5 \%$ and beta $20 \%$, the sample size for each of the intervention and control groups, on the basis of a comparison between two proportions, was 31 patients. With $20 \%$ attrition rate, 36 patients in each group were involved.

\subsubsection{Sampling methods}

Study population was patients that referred to the oncology clinic of Imam Reza Hospital in Mashhad. Patients were randomly selected by interview according to the daily referral list. Those who had primary inclusion criteria received a questionnaire. They were required to fill two sections of it after chemotherapy; otherwise they could not 
be included. Data in this stage was considered as base. Patients at the next course of chemotherapy, after obtaining informed consent, were blindly allocated into two groups.

\subsection{Outcomes}

Number and severity of nausea and vomiting in acute phase (first 24 hours after chemotherapy) were considered as primary outcomes, number and severity of nausea and vomiting in delayed phase (second to fifth day after chemotherapy) were considered as secondary outcomes.

\subsection{Selection criteria}

\subsubsection{Inclusion criteria}

The following were set as the inclusion criteria of the study: Female, Age $\geq 18$ years, clinical diagnosis of breast cancer, history of refractory CINV, normal tests for complete blood count, urea, creatinine, aspartate aminotransferase, alanine aminotransferase, bilirubin, fasting blood sugar, sodium, potassium and at least three courses of chemotherapy remaining.

\subsubsection{Exclusion criteria}

Exclusion criteria of this study were: Total or upper abdominal radiation therapy along with chemotherapy, drugs/therapy for nausea and vomiting not prescribed in this study, hypersensitivity to Sumac or Bunium Persicum, use of Sumac and Bunium Persicum in the seven days prior to the intervention, clinical diagnosis of digestion disorders, non-chemotherapy induced nausea and vomiting, milk allergy, loss of two consecutive or three intermittent doses of Persumac or placebo.

\subsection{Allocation, blinding and randomization}

Laboratory experts used a computerized random number table and allocated patients into two intervention and control groups, and determined coded drug packages for intervention. This was performed in the absence of the research team. The patient was not aware of the type of treatment received by other participants in the study and the analyst was also unaware of the results of the patients. Thus, this study was randomized and double blind.

\subsection{Intervention}

\subsubsection{Regulatory Issues and Study Medication}

Persumac and placebo packaging was similar. All participants received standard regime for CINV: Oral Aprepitant (125 mg on Day 1 and $80 \mathrm{mg}$ on Days 2-3), intravenous Granisetron: $1 \mathrm{mg}$ or $0.01 \mathrm{mg} / \mathrm{kg}$ IV and $2 \mathrm{mg}$ orally on Day 1), and Dexamethasone (12 mg orally or intravenous infusion on Day 1 and $8 \mathrm{mg}$ orally on Days 2-4) (2). Persumac and placebo used three times daily before meals with a glass of cold water; 24 hour before the fifth day of chemotherapy. Patients filled questionnaires at home before bedtime. Crossover was done in the third course of chemotherapy.

\subsubsection{Screening, interventions and evaluations}

Patients were involved in 33 days' study. Participants were randomly divided into two sequences. Sequence AB received first Persumac and then placebo in crossover. Sequence BA received first placebo and then Persumac in crossover. Both sequences received CINV standard therapy in two panels (panell before crossover and panel 2 after crossover). Overall executive steps of study were:

1) Among referred patients for breast cancer to the oncology clinic, those who had at least one chemotherapy course and had inclusion criteria were included in study.

2) The initial assessment of patients (Run- in): Concurrent with the visit of patient for chemotherapy; during interview, study questionnaires were delivered to patients. It required a full explanation for filling out and returning it in the following course.

3) In the second course of chemotherapy, after eligibility qualification and obtaining consent form, patients were randomly allocated into intervention and control groups. In accordance with the study protocol, interventions took place. Questionnaires were delivered to the patients again.

4) Wash out period was six days after the second course of chemotherapy until a day before the third course of chemotherapy.

5) In the third course of chemotherapy in this study, after obtaining the previous questionnaire, patients received crossover interventions. Questionnaires were delivered to the patients again. 


\subsection{Data collection tool}

Outcomes were gathered by Persian questionnaire. Each night, before sleep, patients recorded the data. Number and severity of nausea and vomiting that were measured with self-reporting tool in this three-part questionnaire are as follows:

1) The first part included demographic data, patient's current and historic medical conditions.

2) The second part was a visual analog scale (VAS) - a ten centimeter line scale and a table to record the number/ severity of nausea/ vomiting. Nausea and vomiting had a number on the line between zero to ten (zero $=$ no nausea $/$ vomiting, mild $=1-3$, moderate $=4-6$ severe $=7-9$ and $10=$ very severe).

3) The third part had two questions related to frequency of forgetting our interventions and taking other antiemetic therapies.

The reliability and validity content of this questionnaire has been approved in Najafi et al (2014), Fathi M et al (2014) and Yekta et al (2012) studies. Cronbach's alpha coefficient $=0.948(6,10,11)$.

\subsection{Research ethics}

This research was approved by the Ethics Committee of MUMS (ID number: IR.MUMS.REC.1394.495) on 7 November 2015. Patients were knowingly informed about the study, and signed a Persian consent form, and involvement was entirely voluntary. Participants were authorized to withdraw from the study at their own discretion. All participants' data were confidential. Contact numbers were provided to patients if they had any queries or needed medical help.

\subsection{Bias and confounders}

Period and carry over effects were confounders and rejected by independent-samples t-test. Thus, treatment effect was approved.

\subsection{Statistical analysis}

Data were analyzed by SPSS version 16 (SPSS Inc. Chicago, Illinois, USA). Descriptive statistics were presented as frequencies percent and also by scattering parameters including mean and standard deviation. For treatment effect compared with placebo, crossover analysis by independent sample T-test was used, with calculation of carry over and period effects. Comparison was done between sequence $\mathrm{AB}$ and sequence $\mathrm{BA}$ for assessing of carry over effect, in panel one and panel two. The test was considered significant if level $\leq 0.1$. By $T$ - test, the differences of treatment effect in two sequences compared in two panels. The test was considered significant if level $\leq 0.05$. P value determined. Period effect also calculated, degrees of freedom for the t-statistic is equal to $n-2=67$ and the test was considered significant if level $\leq 0.05$ for period effect.

\subsection{Matherials}

Our Persian traditional medicine remedy includes Sumac (Rhus Coriaria) and Kermani black Zira (Bunium Persicum). Sumac and black zira fruit obtained from a domestic market. Botanists at the Research Institute of Plant Sciences in Ferdowsi University of Mashhad, Iran confirmed Sumac with herbarium number 21021 and Bunium Persicum 28502. Formulation of Persumac preparations was based on Persian traditional medicine texts (9). For this purpose, semi-ground (Jarish) Sumac without the fruit's pit mixed with equal proportion to Jarish of Bunium Persicum by electric mills (Pars Khzar model ML320P). Persumac and placebo similarly packaged and encoded in the School of Persian and Complementary Medicine lab at MUMS. Each dose of Persumac was prepared 2.7 grams into dark sachets. Placebo was lactose, caramel (equal weight) and one drop of black zira essential oil.

\section{Results}

\subsection{Recruitment, Screening, and Completion of intervention}

From October 2015 to May 2016, 134 patients were screened. Ninety-three patients were eligible for study and enrolled randomization and blinding. In panel one, they were allocated randomly into two groups: sequence $\mathrm{AB}(\mathrm{n}=$ 46) for Persumac intervention and sequence BA $(n=47)$ who were assigned to placebo intervention. Thirteen patients left the study in panel one (Five patients had incomplete questionnaires; six used other antiemetic drugs and two were absent in chemotherapy course). Among 80 participants in panel two, Eleven left the study (Five failed to complete the questionnaire, three absent in chemotherapy course and three forgot to take medication. Finally, 35 in sequence $\mathrm{AB}$ and 34 in sequence $\mathrm{BA}$ were eligible for analysis (Figure 1). 


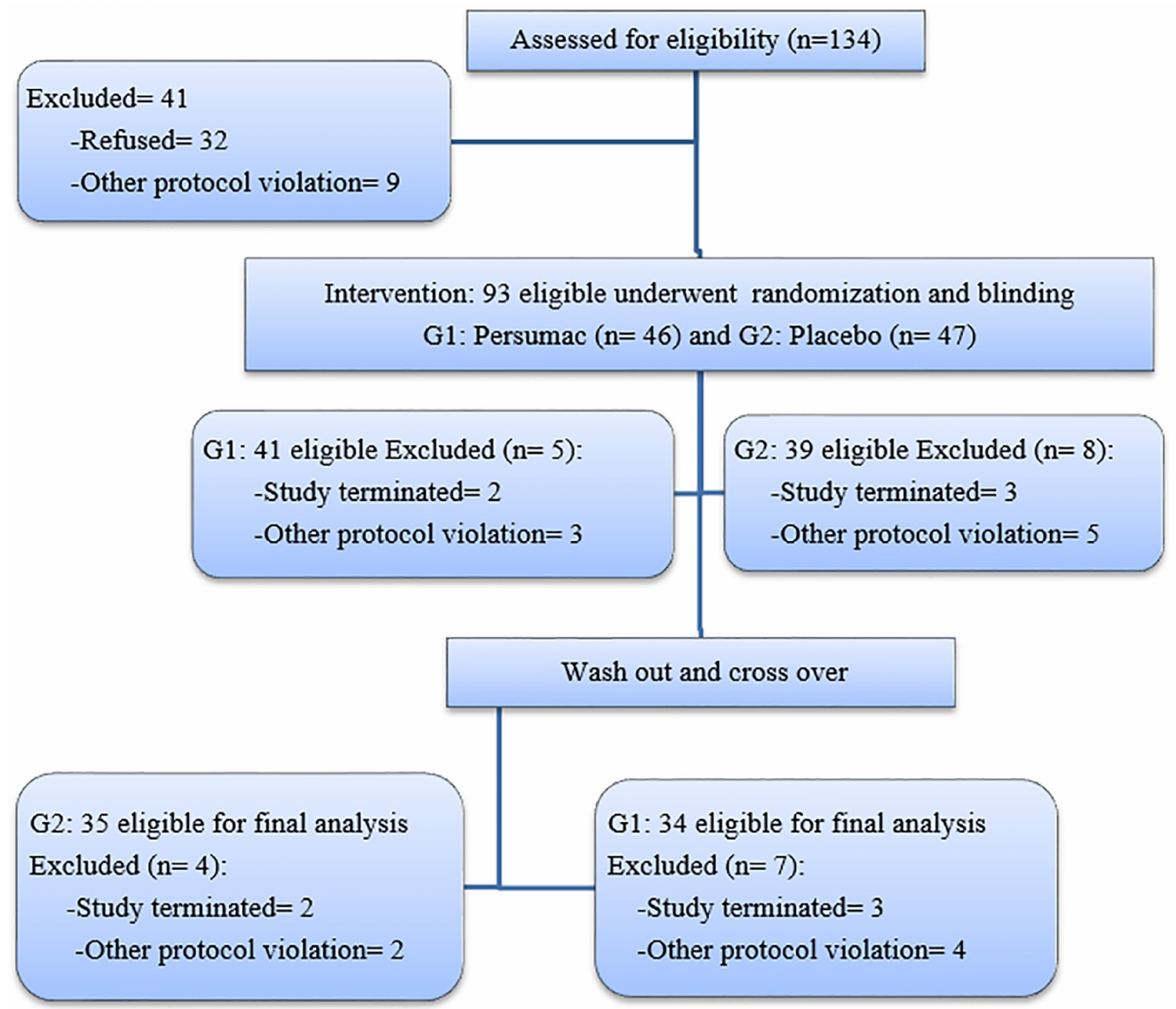

Figure 1. Consort flowchart of study. G1 (sequence $\mathrm{AB}$ ): Intervention at first by Persumac and then Placebo in crossover; G2 (sequence BA): Intervention at first by Placebo and then Persumac in crossover.

\subsection{Demographic findings}

Participants were 55 Iranian, 8 Afghani, 4 Iraqi, 1Pakistani and 1 Tajikistani. 92\% of them were under diploma. Also, in other characters including age, employment and marital status there were no significant differences (Table $1)$.

\subsection{Intervention findings}

Data were gathered in acute and delayed phase. Frequency of nausea and vomiting was between 0-9 per day and severity of nausea and vomiting was between $0-10$. Before intervention, we distributed the questionnaire and had a base from frequency and severity of nausea and vomiting. Results were compared between bases, before and after crossover intervention. According to acquired data, Persumac had significant effect in decrease of frequency and severity of nausea and vomiting. However, in severity of nausea in acute phase, this effect was not significant. Persumac had no verifiable impact on the severity of nausea in acute phase. Intervention data is shown in Table 2. Complete response (no nausea, no vomiting, and no need for rescue therapy) is shown in Table 3. 
http://www.ephysician.ir

Table 1. Demographic data of participants

\begin{tabular}{|c|c|c|c|}
\hline \multicolumn{2}{|l|}{ Characteristics } & Sequence AB & Sequence BA \\
\hline \multirow[t]{3}{*}{ Age (years) } & Median [range] & $47.4[28-61]$ & $46.8[26-64]$ \\
\hline & $25-45$ years $n(\%)$ & $16(47.1)$ & $18(51.4)$ \\
\hline & $>45$ years $n(\%)$ & $18(52.9)$ & $17(48.6)$ \\
\hline \multirow[t]{2}{*}{ Marital status: $\mathrm{n}(\%)$} & Single & $2(6)$ & 0 \\
\hline & Married & $32(94)$ & $35(100)$ \\
\hline \multirow[t]{4}{*}{ Education level: n (\%) } & Illiterate & $11(32)$ & $12(34)$ \\
\hline & Elementary & $15(44)$ & $15(43)$ \\
\hline & Diploma & $7(21)$ & $7(20)$ \\
\hline & Higher & $1(3)$ & $1(3)$ \\
\hline \multirow[t]{2}{*}{ Employment: n (\%) } & Housewife & $27(79)$ & $31(89)$ \\
\hline & Employed & $7(21)$ & $11(4)$ \\
\hline \multirow[t]{2}{*}{ Family history of malignancy: $\mathrm{n}(\%)$} & Yes & $3(9)$ & $3(9)$ \\
\hline & No & $31(91)$ & $32(91)$ \\
\hline \multirow[t]{3}{*}{ Pathology of cancer: $\mathrm{n}(\%)$} & Inductal & $30(88)$ & $29(83)$ \\
\hline & Lobular & $3(9)$ & $5(14)$ \\
\hline & Mixed inductal and lobular & $1(3)$ & $1(3)$ \\
\hline \multirow[t]{4}{*}{ Cancer grade: $\mathrm{n}(\%)$} & I & 0 & 0 \\
\hline & II & $28(82)$ & $32(91)$ \\
\hline & III & $6(18)$ & $3(9)$ \\
\hline & IV & 0 & 0 \\
\hline \multirow[t]{2}{*}{ History of pregnancy vomiting: $\mathrm{n}(\%)$} & Yes & $10(29)$ & $10(28)$ \\
\hline & No & $24(71)$ & $25(72)$ \\
\hline \multirow[t]{2}{*}{ Alcohol use: n (\%) } & Yes & $2(5.9)$ & 0 \\
\hline & No & $32(94.1)$ & $35(100)$ \\
\hline \multirow[t]{2}{*}{ Smoking: n (\%) } & Yes & $3(8.8)$ & $4(11.4)$ \\
\hline & No & $31(91.2)$ & $31(88.6)$ \\
\hline
\end{tabular}

Sequence AB: Intervention by Persumac before crossover and then Placebo after crossover; Sequence BA: Intervention by Placebo before crossover and then Persumac after crossover.

Table 2. Number and severity of nausea and vomiting in three stages

\begin{tabular}{|c|c|c|c|c|c|c|c|c|c|c|c|}
\hline \multirow[t]{2}{*}{ Variable } & \multirow[t]{2}{*}{ Phase } & \multirow[t]{2}{*}{ Sequence } & \multicolumn{3}{|c|}{ Median (Standard Deviation) } & \multicolumn{2}{|c|}{$\begin{array}{l}\text { Carry-over } \\
\text { effect }\end{array}$} & \multicolumn{2}{|c|}{$\begin{array}{l}\text { Period } \\
\text { effect }\end{array}$} & \multicolumn{2}{|c|}{$\begin{array}{l}\text { Treatment } \\
\text { effect }\end{array}$} \\
\hline & & & Base & Panel 1 & Panel 2 & $\mathrm{P}$ & $\mathrm{F}$ & $\mathrm{P}$ & $\mathrm{F}$ & $\mathrm{P}$ & $\mathrm{F}$ \\
\hline \multirow{4}{*}{$\begin{array}{l}\text { Mean N. } \\
\text { of nausea }\end{array}$} & \multirow[t]{2}{*}{ Acute } & $\mathrm{AB}$ & $5.14 \pm 1.94$ & $2.36 \pm 0.86$ & $5.37 \pm 1.49$ & \multirow[t]{2}{*}{0.54} & \multirow[t]{2}{*}{0.5} & \multirow[t]{2}{*}{0.54} & \multirow{2}{*}{0.26} & \multirow{2}{*}{$<0.001$} & \multirow{2}{*}{0.26} \\
\hline & & BA & $5.12 \pm 1.49$ & $5.01 \pm 1.2$ & $2.94 \pm 1.6$ & & & & & & \\
\hline & \multirow[t]{2}{*}{ Delay } & $\mathrm{AB}$ & $3.6 \pm 1.23$ & $2.31 \pm 0.95$ & $3.52 \pm 0.92$ & \multirow[t]{2}{*}{0.15} & \multirow[t]{2}{*}{2.35} & \multirow[t]{2}{*}{0.71} & \multirow[t]{2}{*}{5.9} & \multirow[t]{2}{*}{$<0.001$} & \multirow[t]{2}{*}{5.91} \\
\hline & & BA & $3.34 \pm 1.20$ & $3.15 \pm 1.31$ & $2.22 \pm 1.01$ & & & & & & \\
\hline \multirow{4}{*}{$\begin{array}{l}\text { Mean S. } \\
\text { of nausea }\end{array}$} & \multirow[t]{2}{*}{ Acute } & $\mathrm{AB}$ & $4.83 \pm 1.40$ & $4.54 \pm 2.0$ & $4.15 \pm 0.92$ & \multirow[t]{2}{*}{0.06} & \multirow[t]{2}{*}{0.22} & \multirow[t]{2}{*}{0.62} & \multirow[t]{2}{*}{0.01} & \multirow[t]{2}{*}{0.3} & \multirow[t]{2}{*}{0.01} \\
\hline & & BA & $5.2 \pm 1.11$ & $3.4 \pm 1.52$ & $4.73 \pm 1.71$ & & & & & & \\
\hline & \multirow[t]{2}{*}{ Delay } & $\mathrm{AB}$ & $4.22 \pm 0.88$ & $2.49 \pm 0.73$ & $3.95 \pm 0.74$ & \multirow[t]{2}{*}{0.74} & \multirow[t]{2}{*}{0.21} & \multirow[t]{2}{*}{0.38} & 0.81 & $<0.001$ & 0.8 \\
\hline & & $\mathrm{BA}$ & $4.38 \pm 1$ & $3.88 \pm 0.76$ & 2.66 & & & & & & \\
\hline Mean N. & Acute & $\mathrm{AB}$ & $3.23 \pm 1.72$ & $3.09 \pm 1.46$ & $3.89 \pm 1.59$ & 0.69 & 1.1 & 0.9 & 0.48 & $<0.001$ & 0.48 \\
\hline & & $\mathrm{BA}$ & $3.15 \pm 1.73$ & $3.76 \pm 1.37$ & $2.97 \pm 1.40$ & & & & & & \\
\hline & Delay & $\mathrm{AB}$ & $2.18 \pm 0.80$ & $1.94 \pm 0.67$ & $2.29 \pm 0.68$ & 0.3 & 0.05 & 0.22 & 2.06 & $<0.001$ & 2.06 \\
\hline & & BA & $2.24 \pm 0.74$ & $2.32 \pm 0.65$ & $1.60 \pm 0.71$ & & & & & & \\
\hline Mean S. & Acute & $\mathrm{AB}$ & $5.62 \pm 1.58$ & $3.51 \pm 1.42$ & $4.30 \pm 1.16$ & 0.47 & 0.77 & 0.1 & 0.43 & $<0.001$ & 0.43 \\
\hline & & $\mathrm{BA}$ & $4.75 \pm 1.55$ & $4.81 \pm 1.26$ & $3.31 \pm 1.15$ & & & & & & \\
\hline & Delay & $\mathrm{AB}$ & $4.27 \pm 1.25$ & $2.20 \pm 0.74$ & $4.18 \pm 0.99$ & 0.84 & 1.86 & 0.38 & 1.03 & $<0.001$ & 1.03 \\
\hline & & $\mathrm{BA}$ & $3.49 \pm 0.94$ & $4.09 \pm 1.09$ & $2.35 \pm 0.63$ & & & & & & \\
\hline
\end{tabular}

Base: without intervention; Sequence AB: Intervention by Persumac before crossover and then Placebo after crossover; Sequence BA: Intervention by Placebo before crossover and then Persumac after crossover; Panel 1: Intervention before crossover, Panel 2: Intervention after crossover, N: Number, S: Severity, P: Alpha error, F: degree of freedom. 
Table 3. complete response

\begin{tabular}{|l|l|l|l|l|}
\hline $\begin{array}{l}\text { Complete Response } \\
\text { n (\%) }\end{array}$ & Phase & Sequence & Panel 1 & Panel 2 \\
\cline { 2 - 5 } & Acute & AB & $5(15)$ & 0 \\
\cline { 2 - 5 } & & BA & $1(3)$ & $6(17)$ \\
\cline { 2 - 5 } & Delay & AB & $9(27)$ & $1(3)$ \\
\cline { 3 - 5 } & & BA & 0 & $8(23)$ \\
\hline
\end{tabular}

Sequence AB: Intervention by Persumac before crossover and then Placebo after crossover; Sequence BA: Intervention by Placebo before crossover and then Persumac after crossover, Panel 1: Intervention before crossover; Panel 2: Intervention after crossover.

\section{Discussion}

Based on the obtained results; Persumac had a significant effect in CINV. The exception was severity of nausea in acute phase $(P=0.3)$, because carry over effect was not significant $(p=0.06)$. Therefore, the impact of Persumac on severity of nausea in acute phase cannot be verified. Although the number of complete response to treatment were not a significant percentage, differences between Persumac intervention in acute and delayed phase is significant and it can be said that Persumac enhances the control of CINV. The Persumac dose in Persian Medicine textbooks is 13 grams daily for adults (9) but according to the WHO guideline for methodologies on research and evaluation of traditional medicine (12); administered dosage was reduced to 8 gram/day. It seems probable because Persumac is affectless on severity of nausea in acute phase. Perhaps, the acute phase nausea may be controlled, if the prescribed dose was 13 gram/day versus 8 grams daily. Also, in a search of scientific databases ISI, Pub Med, Scopus, SID (Scientific Iranian Database) and Embase, no study with Persumac or similar materials for CINV were found. Fathi et al. (2014), in a study about the effects of Body Position, reported significant effect on the number and severity of nausea and vomiting in acute and delayed phase. Compared to Persumac, it was effective on severity of nause a in the acute phase. This study was a single-blind without crossover. Patients had different types of cancer, mostly in the digestive system. They involved different chemotherapy regimens and both sexes participated in the study (10). Eghbali et al. (2016), in a study on the effect of acupressure in breast cancer patients, showed a significant reduction in the number and severity of acute and delayed nausea and vomiting. The study was performed in 24 experimental and control groups. In clinical view, number of nausea and vomiting decreased more than Persumac. The measuring tool was different (Morrow) and patients included non-refractory CINV (13). Compared with Persumac, reported effects of cardamom aromas on CINV in a Khalili et al. (2014) study, were less. This research was in the acute phase of CINV and patients with different types of cancer and chemotherapy regimens and in both sexes (14). In clinical trial with patients who had different types of cancer and chemotherapy regime, in both sexes and conducted without crossover; Volatile oil of Mentha spicata and Mentha piperita in a study by Tayarani-Najaran et al (2013), had better effect, especially in acute phase (15). In a Sana'ti F. et al (2014) study, triple blind, no crossover, with 43 patients, the effect of ginger on frequency and severity of nausea and vomiting in acute phase was similar to Persumac (6). Panahi et al. reported that ginger decreased the number of nausea in acute phase and had no effect in delayed phase (16). In a report by Fatma Genc and Mehtap Tan (2014), a quasi-experimental model with control group, in local center- acupuncture in P6, had significant effect on number and severity of nausea in delayed phase and number and severity of vomiting in acute phase the second day after chemotherapy, and was better than Persumac (17). In a Junya Sato et al (2016) research, the number and severity of nausea and vomiting in chemotherapy with and without olanzapine was assessed in 45 participants. Olanzapine had better effect on number and severity of nausea and vomiting and complete response rate. But Persumac was free from olanzapine complications such as weight gain, somnolence, sleeplessness, constipation, akathisia and elevated triglyceride levels (18). Mechanism of Persumac on CINV is a field of new research. Majority of Sumac is composed of tannins. Seventy-four types of hydrolysable tannins derivatives have been identified, and have astringent and tonic effects (19-21). Forasmuch as free radicals induced by chemotherapy can cause serotonin release from the enterochromaffin cells in small intestine (22) and also Rhus Coriaria and Bunium Persicum are potent antioxidant and effect on hyperactive gut ( 20-21), perhaps Persumac by this way can impact on CINV. In Persian medicine, Sumac and Bunium Persicum are astringent and the tonic effects the stomach. (23-25). According to Iran's health ministry rules, traditional remedies shape and way of use in clinical trials must comply with the Persian medicine texts. Patients did not like the Persumac form and shape. Maybe extraction and preparation of suitable pharmaceutical form have better effects. Cheap, convenient access and simple methods of preparation and use are preferences of Persumac, compared to other options. Regarding the limitation of study, we should say that consumption of all drugs were not under supervision of the researcher. Course commencement and cycle of chemotherapy was different. 


\section{Conclusions}

In summary, the findings of this study showed that Persumac in patients with refractory CINV may be helpful, and increase the continuation of chemotherapy in patients. The applicable importance of this research is the potential of adding an available, cheap option for therapist in refractory CINV. Additional research in this field with dose modification, various cancers, and patients with more versatile features, more complete methodology and assessment tools, could be the right direction for future research on this topic.

\section{Trial registration:}

This trial was registered at the Clinical Trials.gov ID: NCT02787707.

\section{Acknowledgments and funding:}

This study is part of a Ph.D. thesis and under grant; No: 930735 of Research Chancellery of MUMS. We would also like to thank the participants, from Imam Reza Hospital; oncology clinic staff, library staff at the Persian and Complementary Medicine faculty and Imam Reza Hospital at MUMS, and laboratory staff at Persian and Complementary Medicine faculty for all their patience and tolerance.

\section{Conflict of Interest:}

There is no conflict of interest to be declared.

\section{Authors' contributions:}

All authors contributed to this project and article equally. All authors read and approved the final manuscript.

\section{References:}

1) Navari RM. Management of Chemotherapy-Induced Nausea and Vomiting: New Agents and New Uses of Current Agents. 1 ed. Springer; 2016. doi: 10.1007/978-3-319-27016-6.

2) DeVita VT, Lawrence TS, Rosenberg SA. Cancer: principles \& practice of oncology. 10th ed. Philadelphia: Lippincott Williams \& Wilkins. 2014; 1107-52, 976-82.

3) Hocking CM, Kichenadasse G. Olanzapine for chemotherapy-induced nausea and vomiting: a systematic review. Support Care Cancer. 2014; 22(4): 1143-51. doi: 10.1007/s00520-014-2138-y. PMID: 24522741.

4) Abe M, Kasamatsu Y, Kado N, Kuji S, Tanaka A, Takahashi N, et al. Efficacy of Olanzapine Combined Therapy for Patients Receiving Highly Emetogenic Chemotherapy Resistant to Standard Antiemetic Therapy. Biomed Res Int. 2015; 2015: 956785. doi: 10.1155/2015/956785. PMID: 26425564, PMCID: PMC4573881.

5) Vanaki Z, Matourypour P, Gholami R, Zare Z, Mehrzad V, Dehghan M. Therapeutic touch for nausea in breast cancer patients receiving chemotherapy: Composing a treatment. Complement Ther Clin Pract. 2016; 22: 64-8. doi:10.1016/j.ctcp.2015.12.004. PMID: 26850808.

6) Sana'ti F, Najafi S, Kashaninia Z, Naseri M, Hoseinzadeh S. Effect of Ginjer on chemotherapy induced acute nausea and vomiting (persian). Iranian Journal of Breast Diseases. 2014; 25(1): 7-14.

7) Nazari M, Taghizadeh A, Orafaei H, Rakhshandeh H, Bazzaz MM, Shokri J, et al. Nausea and vomiting in Iranian Traditional Medicine based on Avicenna's viewpoint. Electronic physician. 2015; 7(2): 1047-53. doi: 10.14661/2015.1047-1053. PMID: 26120413, PMCID: PMC4477764.

8) Aliasl F, Ranjbar pazuki M, Sadeghpour O, Mohammadi A, Minaee MB, Aliasl J, et al. Eeffective herbal medicines on Nausea and vomiting based on Canon of Avecina. Advances in Environmental Biology. 2015; 9(3): 557-60.

9) Tonekaboni MM. Tohfatol mo'menin (persian). 1, 2. Qom: Noor e vahy. 2011; 56, 443, 504.

10) Fathi M, Nikbakht Nasrabadi A, Valiee S. The effects of body position on chemotherapy-induced nausea and vomiting: a single-blind randomized controlled trial. Iran Red Crescent Med J. 2014; 16(6): e17778. doi: 10.5812/ircmj.17778. PMID: 25068049, PMCID: PMC4102982.

11) Ebrahimi SM, Parsa Yekta Z, Nikbakht-Nasrabadi A, Hosseini M, Sedighi S, Salehi Surmaghi MH. Assessment of Palliative Effects of Ginger on Chemotherapy-Induced Nausea: Delayed Phase. Journal of Isfahan Medical School. 2012; 30(219): 2324-32.

12) $\mathrm{WHO} / \mathrm{EDM} / \mathrm{TRM} / 2000.1$, General guidelines for methodologies on research and evaluation of traditional medicine. Geniva: World Health Organization; 2000.

13) Eghbali M, Yekaninejad MS, Varaei S, Jalalinia SF, Samimi MA, Sa'atchi K. The effect of auricular acupressure on nausea and vomiting caused by chemotherapy among breast cancer patients. Complement Ther Clin Pract. 2016; 24: 189-94. doi: 10.1016/j.ctcp.2016.06.006. PMID: 27502820. 
14) Khalili Z, Khatiban M, Faradmal J, Abbasi M, Zeraati F, Khazaei A. Effect of Cardamom aromas on the Chemotherapy-induced Nausea and Vomiting in Cancer Patients. Scientific Journal of Hamadan Nursing \& Midwifery Faculty. 2014; 22(3): 64-73.

15) Tayarani-Najaran Z, Talasaz-Firoozi E, Nasiri R, Jalali N, Hassanzadeh M. Antiemetic activity of volatile oil from Mentha spicata and Mentha $\mathrm{x}$ piperita in chemotherapy-induced nausea and vomiting. Ecancermedicalscience. 2013; 7: 290. doi: 10.3332/ecancer.2013.290. PMID: 23390455, PMCID: PMC3562057.

16) Panahi Y, Saadat A, Sahebkar A, Hashemian F, Taghikhani M, Abolhasani E. Effect of ginger on acute and delayed chemotherapy-induced nausea and vomiting: a pilot, randomized, open-label clinical trial. Integr Cancer Ther. 2012; 11(3): 204-11. doi: 10.1177/1534735411433201. PMID: 22313739.

17) Genc F, Tan M. The effect of acupressure application on chemotherapy-induced nausea, vomiting, and anxiety in patients with breast cancer. Palliat Support Care. 2015; 13(2): 275-84. doi: 10.1017/S1478951514000248. PMID: 24787745.

18) Sato J, Kashiwaba M, Komatsu H, Ishida K, Nihei S, Kudo K. Effect of olanzapine for breast cancer patients resistant to triplet antiemetic therapy with nausea due to anthracycline-containing adjuvant chemotherapy. Jpn J Clin Oncol. 2016; 46(5): 415-20. doi: 10.1093/jjco/hyw011. PMID: 26951840.

19) Abu-Reidah IM, Ali-Shtayeh MS, Jamous RM, Arráez-Román D, Segura-Carretero A. HPLC-DAD-ESIMS/MS screening of bioactive components from Rhus coriaria L. (Sumac) fruits. Food chem. 2015; 166: 179-91. doi: 10.1016/j.foodchem.2014.06.011. PMID: 25053044.

20) Jalilzadeh-Amin G, Maham M, Dalir-Naghadeh B, Kheiri F. Effects of Bunium persicum (Boiss.) Essential oil on the contractile responses of smooth muscle (An in vitro Study). Veterinary Research Forum; 2011; 2(2): 87-96.

21) Janbaz KH, Shabbir S, Mehmood MH, Gilani AH. Pharmacological basis for the medicinal use of rhus coriaria in hyperactive gut disorders. Bangladesh Journal of Pharmacology. 2014; 9(4): 636-44. doi: 10.3329/bjp.v9i4.20879.

22) Aapro M, Jordan K, Feyer P. Prevention of Nausea and Vomiting in Cancer Patients. london: Springer. 2013; 1-55. doi: 10.1007/978-1-907673-58-0.

23) Aghsaraee J. Sharh-e-Aghsaraee(Hall ol Mujazz) (Arabic). In: Isfahani MM, editor. 1. Tehran: Iran University of Medical Sciences. 2008; 209.

24) Ibne-Nafis. A'shshamel fes senaa'tot tebbiah(arabic). 15. 1 ed. Tehran: Research Institute for Islamic and Complementary Medicine (RICM). 2000; 620-7.

25) Ibne-Nafis. A'shshamel fes sena'to tebbiah(arabic). 25. 1 ed. Tehran: Research Institute for Islamic and Complementary Medicine (RICM). 2000; 416. 